DOI: https://doi.org/10.22206/cyap.2019.v2i2.pp7-28

\title{
Proporción de costo total del aCero dentro de los Costos DIRECTOS DE PROYECTOS DE VIVIENDAS EN LA PROVINCIA DE Santo Domingo (República Dominicana) para el año 2019
}

\author{
Steel cost proportion with respect to direct costs in housing projects in Santo \\ Domingo (Dominican Republic) for year 2019
}

\section{Tulio Rodríguez ${ }^{\mathrm{a}}$ y Marleni Ventura ${ }^{\mathrm{b}}$}

Recibido: 29/10/19 • Aprobado: 16/12/19

Cómo citar: Rodríguez, T. R., \& Ventura, M. (2019). Proporción de costo total del acero dentro de los costos directos de proyectos de viviendas en la provincia de Santo Domingo (República Dominicana) para el año 2019. Ciencia, Ingenierias y Aplicaciones, 2(2), 7-28. Doi: https:/doi.org/10.22206/cyap.2019.v2i2.pp7-28

\section{Resumen}

Este trabajo de investigación tiene como objetivo determinar la proporción de costo total del acero dentro de los costos directos de proyectos de viviendas de clase económica, media y alta en la provincia de Santo Domingo, República Dominicana, para el año 2019. Para realizar el cálculo de dicho indicador se tomaron como muestras presupuestos de cada tipo de vivienda, a partir de los cuales se homogenizó el costo directo de acero para luego ser llevado a valor presente. Este documento presenta los antecedentes y la situación actual del objeto de estudio, la metodología utilizada para la selección de la muestra, la recopilación de datos y su posterior análisis. Además, se incluye una descripción de los proyectos seleccionados como casos de estudio, los resultados obtenidos, y las conclusiones obtenidas a partir de los mismos.

Palabras clave: costo de acero; análisis de costos; viviendas; administración de la construcción; construcción.

\begin{abstract}
The objective of this work is to estimate the steel cost proportion with respect to direct costs in housing construction for low, middle, and high economic classes

\footnotetext{
a Instituto Tecnológico de Santo Domingo (INTEC), Santo Domingo, República Dominicana Correo-e: tulio.rodriguez@intec.edu.do

b Instituto Tecnológico de Santo Domingo (INTEC), Santo Domingo, República Dominicana

Correo-e: marleny.ventura@gmail.com
}

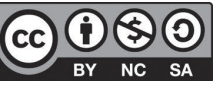


in Santo Domingo, Dominican Republic, for the year 2019. To perform the calculations of this indicator a sample of the budgets for each type of construction was taken, followed by the homogenization of the steel direct costs. This document presents a literature review and the current state of this topic, the methodology employed to select a sample, the process to obtain and analyze the data. Moreover, a description of the projects selected for the case study is presented, followed by the results and the conclussions.

Keywords: Steel cost; cost analysis; housing; construction management; construction. 


\section{Introducción}

Estudios recientes muestran que la República Dominicana presenta altos valores de déficit habitacional en todos los rangos sociales (División Investigación ONE, 2010: Ministerio de Economía, Planificación y Desarrollo, 2018). El Estado Dominicano e instituciones asociadas han trabajado para reducir este margen incentivando a actores del sector por medio de promulgaciones y acuerdos a la construcción de viviendas. Según datos de la Asociación Dominicana de Constructores y Promotores de Viviendas (ACOPROVI) y el Banco Central, en el primer trimestre de 2018 la construcción de viviendas en República Dominicana creció un $9,3 \%$ reflejando un aumento en el aporte de esta actividad al Producto Interno Bruto (PIB) de un $12 \%$ con referencia al 2007.

Del área total de construcción registrada, el $86.8 \%$ correspondió al área de construcción vendible, lo cual equivale a 4, 494,718.3 m². La vivienda fue el principal destino, con un $88.0 \%$ de área de construcción vendible, equivalente a $3,954,884.3 \mathrm{~m}^{2}$, siguiendo, en menor medida, los destinos comercio $\left(231,474.7 \mathrm{~m}^{2}: 5.1 \%\right)$, oficina $\left(114,781.3 \mathrm{~m}^{2}: 2.6 \%\right)$, y educación (109,327.0 m²: $2.4 \%)$ (ONE, 2019).Esta construcción de viviendas se ha dirigido a todos los rangos de adquisición económica en viviendas de bajo costo y torres de apartamentos diseñadas acorde a los requerimientos estructurales y arquitectónicos establecidos por los reglamentos M-009, Especificaciones Generales para la Construcción de Edificaciones, y M-033, Reglamento para Diseño y Construcción de Estructuras en Hormigón Armado. Estos requerimientos estructurales están regidos por el código ACI-318 para hormigón armado reforzado, siendo este último una de las bases que definen los costos directos en la construcción de viviendas. Para el objeto de estudio de esta investigación nos centraremos en el acero reforzado, insumo básico del hormigón armado, y su representación proporcional dentro de los costos directos en la construcción de viviendas. 
En la actualidad, en la República Dominicana existe el Índice de Costos Directos de la Construcción de Viviendas (ICDV) que elabora la ONE con apoyo de ACOPROVI. El Índice de Costos Directos de la Construcción de Viviendas (ICDV) es una herramienta estadística que permite conocer las variaciones mensuales que experimenta el costo de la construcción de cuatro tipos de viviendas: unifamiliar de 1 y 2 niveles, y multifamiliar de 4 y 8 niveles o más, en el Distrito Nacional y la provincia de Santo Domingo (se excluyen los costos indirectos como: terreno, diseño, permisos de construcción, costos financieros, beneficios de la empresa constructora, etc.). Su metodología de cálculo responde a la del Índice de Laspeyres, el cual contempla una canasta con estructura de ponderaciones fijas en el tiempo y compara los precios del período de referencia con los del período base (octubre del 2009). Este índice permite conocer las varicaciones del precio en el tiempo de cada uno de estos proyectos, pero no proporciona información de cuánto representa cada insumo dentro de este costo total. La Cámara Dominicana de la Construcción elabora el Índice de Precios de Edificaciones Habitacionales que permitirá medir las variaciones en los precios de los insumos de la construcción. No se trata de la estructura de costos para hacer la estimación del valor de mercado de las edificaciones al presente, sino para realizar ajustes o enmiendas a partidas específicas en los presupuestos al momento de ser cubicadas, considerando las variaciones en los precios. Esta incluye el insumo acero, pero igual al índice anterior no permite identificar qué porcentaje representa el acero del costo total del proyecto.

En otros países de Latinoamérica los índices de precios de la construcción incluyen un análisis de costo por cada partida y su porcentaje de participación en el costo total. Si vemos el caso de Uruguay, que a través del Instituto Nacional de Estadística (I.N.E.) elabora y publica Índice de Costo de la Construcción, este informe incluye: 
Cuadro 1 - Ponderaciones por tipo de obra

\begin{tabular}{|c|c|c|c|c|c|}
\hline \multirow{2}{*}{ Rubros de obra } & \multirow{2}{*}{$\begin{array}{l}\text { Todos } \\
\text { los } \\
\text { tipos }\end{array}$} & \multicolumn{4}{|c|}{ Tipos de obra } \\
\hline & & 1 & 2 & 3 & 4 \\
\hline TOTAL & 100,00 & 100,00 & 100,00 & 100,00 & 100,00 \\
\hline $\begin{array}{l}01 \text { - Repl. e Implantación } \\
02 \text { - Hormigón armado } \\
03 \text { - Albañilería }\end{array}$ & $\begin{array}{r}0,56932 \\
11,25999\end{array}$ & $\begin{array}{r}0,58511 \\
13,62852\end{array}$ & $\begin{array}{r}0,27803 \\
13,08884\end{array}$ & $\begin{array}{l}0,70618 \\
8,51713\end{array}$ & $\begin{array}{l}0,45099 \\
6,83302\end{array}$ \\
\hline $03.1-$ Muros & 7,62788 & 7,52506 & 5,88049 & 9,05904 & 6,50513 \\
\hline 03.2 - Revoques & 5,28965 & 5,90065 & 5,48892 & 4,01995 & 5,33048 \\
\hline 03.3 - Contr., pisos y zócalos & 4,76004 & 5,80861 & 3,54287 & 3,68925 & 3,81391 \\
\hline 03.4- Otros rubros albañilería & 7,23812 & 3,94349 & 8,20304 & 9,09283 & 14,72305 \\
\hline 04 - Carpintería & 1,73886 & 2,07842 & 1,25383 & 1,68297 & 0,96592 \\
\hline 05 - Herrajes & 0,21892 & 0,22559 & 0,21603 & 0,20507 & 0,22251 \\
\hline 06 - Herrería & 0,62021 & 0,74494 & 0,64787 & 0,52533 & 0,34098 \\
\hline 07 - Aluminio & 1,50776 & 1,47903 & 2,35563 & 1,52630 & 1,04193 \\
\hline 08 - Cortinas de enrollar & 0,69978 & 1,03745 & 0,51159 & 0,50339 & \\
\hline 09 - Instalaciones eléctricas & 2,74390 & 2,21671 & 3,83903 & 2,07170 & 5,09540 \\
\hline 10 - Instalaciones sanitarias & 4,70019 & 3,11946 & 5,35097 & 6,96376 & 5,70083 \\
\hline 11 - Aparatos y grifería & 1,10447 & 0,92072 & 1,21098 & 1,38195 & 1,17555 \\
\hline 12 - Ascensor & 1,37360 & 2,73082 & & & \\
\hline 13 - Vidrios & 0,34572 & 0,34041 & 0,23431 & 0,46578 & 0,21795 \\
\hline 14 - Pinturas & 2,86588 & 3,18310 & 2,60219 & 2,90341 & 1,86339 \\
\hline 15 - Gastos generales & 10,22949 & 10,56284 & 9,88664 & 9,86967 & 9,93598 \\
\hline 16 - Impuesto valor agregado & 14,92557 & 15,18712 & 14,85599 & 14,53225 & 14,76992 \\
\hline 17 - Leyes sociales & 19,26348 & 17,94373 & 19,70395 & 21,20324 & 20,07364 \\
\hline 18 - Conexiones definitivas & 0,59495 & 0,48668 & 0,52182 & 0,76453 & 0,71119 \\
\hline 19 - Permisos construcción & 0,32222 & 0,35152 & 0,32699 & 0,31629 & 0,22822 \\
\hline
\end{tabular}

Figura 1. Ponderaciones por tipo de obra

Fuente: metodología - Base diciembre 1999=100 Índice de costos de la construcción (INE). Tipo 1: torre, varios niveles con ascensor, económica o mediana. Tipo 2: viviendas en planta baja y 3 niveles sin ascensor, económicas en uno o más bloques sin infraestructura propia.

Podemos observar que para los proyectos tipo 1 y 2 el hormigón armado representa $13 \%$ aproximadamente, esta partida incluye el acero.

En el caso de Argentina el Consejo Profesional de Arquitectura y Urbanismo recopila los últimos valores publicados de los índices y modelos de costos de la construcción para la Ciudad de Buenos Aires y el Gran Buenos Aires. Esta recopilación se hace mensual y en la misma se presenta el índice de la Revista Vivienda. 


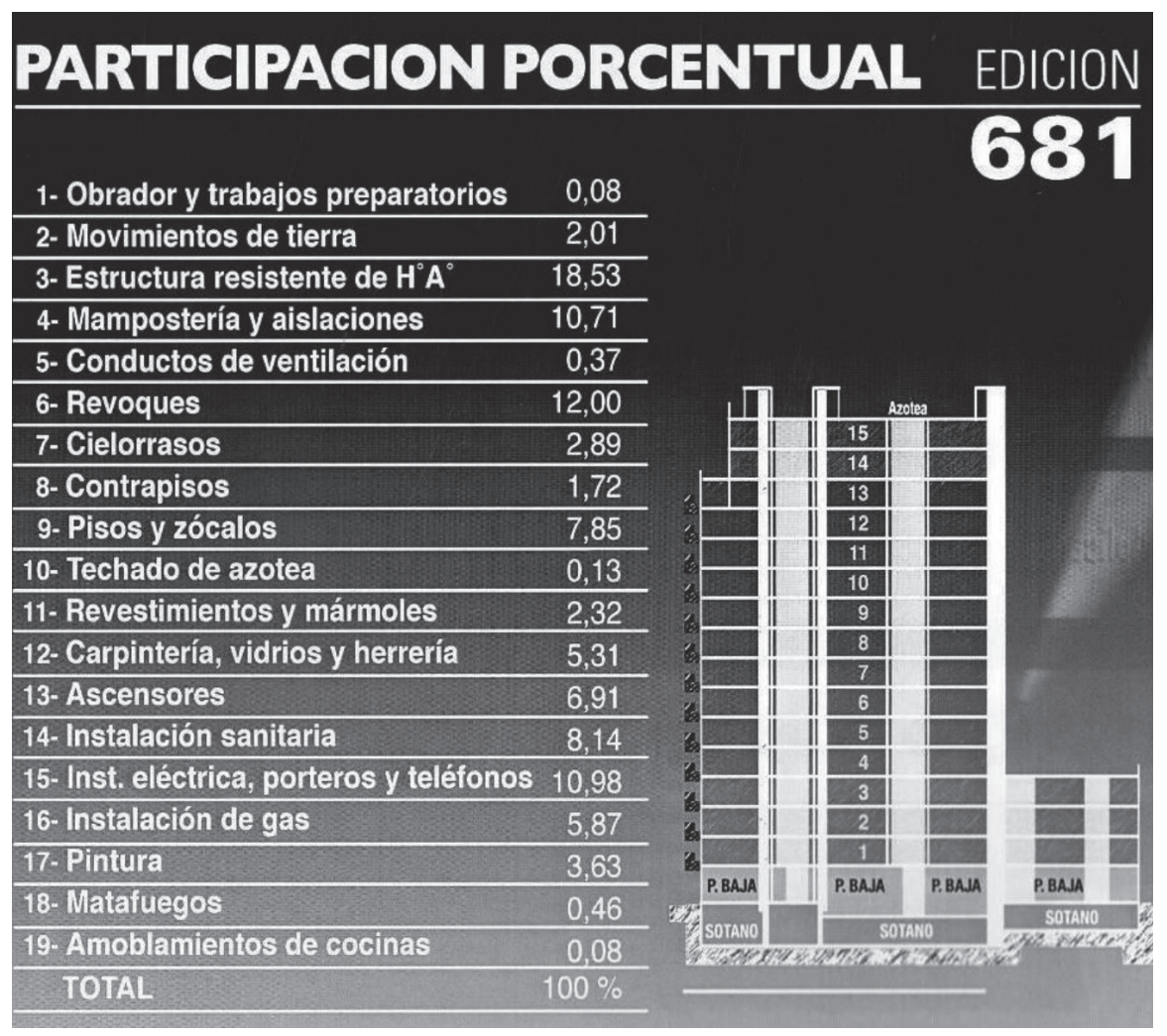

Figura 2. Índice de la Revista Vivienda. No. 681 (abr. 2019). Inserto entre p. 114 y 115.

Aquí se puede observar cómo para esta edificación de 15 niveles el costo porcentual de la estructura resistente de H.A. el costo es de $18.53 \%$. Este costo también incluye el acero.

\section{Herramientas y métodos}

\subsection{Diseño de la Investigación}

El estudio de caso es un tipo de estrategia metodológica que tiene como objetivo el estudio a profundidad de un fenómeno, situado en una unidad de análisis particular, la cual forma parte de un universo poblacional. Este llamado "caso" es conocido como el sistema integral y particular con el interactúa el objeto de estudio, el cual puede ser desde 
una empresa, o institución, hasta una persona o grupo (Bernal, 2010). Para llevar a cabo esta investigación se utilizó el estudio de caso múltiple, tomando en cuenta que el análisis del objeto de estudio se situará en proyectos de viviendas específicos.

\subsection{Cantidad y tipo de muestra}

Para la selección de la muestra se utilizó el siguiente esquema, recomendado por Kinnear y Taylor (1993):

- Definir la población: en este caso la población a la que pertenece el objeto de estudio son los proyectos de viviendas ubicados en la provincia de Santo Domingo.

- Identificar el marco muestral: en este caso, el marco muestral utilizado fue los presupuestos para proyectos de vivienda, realizados por empresas locales en la provincia de Santo Domingo.

- Elegir un procedimiento de muestreo: para el desarrollo de esta investigación se utilizó un muestro no probabilístico. Dicho muestreo consistió en identificar personas, instituciones y/o grupos clave que nos facilitaron el acceso a la data, o a otras personas claves que finalmente nos otorgaron acceso a la muestra deseada.

- Determinar el tamaño de la muestra:

Para la selección de la muestra, la población fue divida en tres estratos o subgrupos de la siguiente manera:

- Proyectos de viviendas de clase económicas: viviendas de hasta cuatro (4) niveles construidas con formaleta.

- Proyectos de viviendas de clase media: torres inmobiliarias entre ocho (8), diez (10) y doce (12) niveles.

- Proyectos de viviendas de clase alta: torres inmobiliarias de más de 12 niveles.

En este caso el tamaño de muestra definido por cada subgrupo fue de cinco (5) proyectos, basado en la disponibilidad de recursos 
y accesibilidad de la información obtenida a través del muestreo de "bola de nieve".

- Seleccionar la muestra.

\subsection{Homogenización de la muestra}

En busca de controlar la variable del método de análisis utilizado para la cuantificación del armado dentro de los presupuestos elegidos como muestra, se realizó un nuevo cálculo de este, bajo el método de optimización, considerando el acero figurado. La selección de dicho método fue fundamentada en los resultados obtenidos de la investigación "Análisis comparativo entre el método de asignación directa y el de optimización para cuantificación del refuerzo levantado en los apoyos en placas sólidas y su efecto en la generación de desperdicios" presentada por Rodríguez y Abbott (2008).

Para el cálculo del valor presente de los costos directos planteados en los presupuestos de muestra se utilizó el Índice de Costos Directos de la Construcción de Viviendas (ICDV), elaborado por la Oficina Nacional de Estadística para representar costos fuera del rango de los últimos seis (6) meses del presente año.

\subsection{Análisis de la muestra y evaluación de resultados}

Luego de la homogenización de la data recopilada se tomaron los valores resultantes para calcular una media ponderada utilizando el modelo PERT. Este modelo consiste en una media ponderada considerando los valores extremos, el mayor y el mínimo, junto a la suma del valor promedio multiplicado por cuatro dividido entre seis.

$$
\text { Mpond. }=\frac{\text { Valor menor }+4 * \text { valor promedio }+ \text { valor mayor }}{6}
$$

La importancia relativa de los valores se calculará considerando los costos totales de las variables "costo de acero" y "costo directo" por tipo de construcción de vivienda. 


\section{Análisis de los resultados}

\subsection{Descripción de las muestras seleccionadas}

3.1.1. Tipo 1 (Proyectos de viviendas de clase económica):

En la tabla 1 se presenta un resumen de las principales características de las muestras seleccionadas como vivienda tipo 1 (Viviendas de clase económica). En adición, la tabla 2 refleja el área de construcción de cada muestra del Tipo 1.

Tabla 1. Detalle muestra viviendas económicas (Tipo 1)

\begin{tabular}{|l|l|}
\hline Tipo de Muestra: & Vivienda económica de cuatro niveles \\
\hline Terminación: & Económica \\
\hline Sistema Estructural: & Zapatas corridas con muros en formaleta y refuerzos en malla \\
\hline $\begin{array}{l}\text { Muestras: M1, M3, } \\
\text { M4: }\end{array}$ & Refuerzos adicionales en conexión muro-entrepiso \\
\hline Muestras M2, M5: & Refuerzos adicionales mínimos (en áreas críticas y esquinas) \\
\hline
\end{tabular}

Tabla 2. Detalle área de construcción proyectos Tipo 1

\begin{tabular}{|c|c|}
\hline Muestra & Área de construcción $\left(\boldsymbol{M}^{2}\right)$ \\
\hline T1 M1 & 479.68 \\
\hline T1 M2 & 398.27 \\
\hline T1 M3 & 611.72 \\
\hline T1 M4 & 987.13 \\
\hline T1 M5 & 597.58 \\
\hline
\end{tabular}

\subsubsection{Tipo 2 (Proyectos de viviendas de clase media)}

A continuación, se presenta los detalles de las muestras seleccionadas como vivienda tipo 2 (Viviendas de clase media), incluyendo su área de construcción. 
Tabla 3. Detalle muestra viviendas de clase media (Tipo 2)

\begin{tabular}{|c|c|c|c|c|c|}
\hline $\begin{array}{l}\text { Tipo de } \\
\text { Muestra: }\end{array}$ & \multicolumn{5}{|c|}{ Vivienda clase media de ocho a doce niveles } \\
\hline Terminación: & \multicolumn{5}{|c|}{ Media - Alta } \\
\hline $\begin{array}{l}\text { Sistema } \\
\text { Estructural: }\end{array}$ & \multicolumn{5}{|c|}{ Sistema aporticado con losas aligeradas y losas macizas } \\
\hline No. Muestra & M1 & M2 & M3 & M4 & M5 \\
\hline Niveles & $\begin{array}{l}2 \text { sótanos } \\
10 \text { niveles }\end{array}$ & $\begin{array}{l}2 \text { sótanos } \\
10 \text { niveles }\end{array}$ & $\begin{array}{l}1 \text { sótano } \\
8 \text { niveles }\end{array}$ & 10 niveles & $\begin{array}{c}1 \text { sótano } \\
11 \text { niveles }\end{array}$ \\
\hline $\begin{array}{l}\text { Área de } \\
\text { construcción }\end{array}$ & $\begin{array}{c}16,393.10 \\
\mathrm{~m}^{2}\end{array}$ & $\begin{array}{c}6,567.78 \\
\mathrm{~m}^{2}\end{array}$ & $\begin{array}{c}3,483.03 \\
\mathrm{~m}^{2}\end{array}$ & $\begin{array}{c}5,051.30 \\
\mathrm{~m}^{2}\end{array}$ & $6,058.36 \mathrm{~m}^{2}$ \\
\hline Fundaciones & \multicolumn{4}{|c|}{ Zapatas aisladas con vigas riostras y zapatas corridas } & $\begin{array}{l}\text { Platea de } \\
\text { fundación }\end{array}$ \\
\hline
\end{tabular}

\subsubsection{Tipo 3 (Proyectos de viviendas de clase alta)}

En la tabla 4 se presenta el detalle de las principales características de las muestras seleccionadas como vivienda tipo 2 (Viviendas de clase alta), incluyendo el área de construcción:

Tabla 4. Detalle muestra viviendas de clase alta (Tipo 3)

\begin{tabular}{|c|c|c|c|c|c|}
\hline Tipo de Muestra: & \multicolumn{5}{|c|}{ Vivienda clase alta de doce niveles o más } \\
\hline Terminación: & \multicolumn{5}{|l|}{ Alta } \\
\hline $\begin{array}{l}\text { Sistema } \\
\text { Estructural: }\end{array}$ & \multicolumn{5}{|c|}{ Sistema aporticado con losas aligeradas y losas macizas } \\
\hline No. Muestra & T3 M1 & T3 M2 & T3 M3 & T3 M4 & T3 M5 \\
\hline Niveles & $\begin{array}{l}4 \text { sótano } \\
15 \text { niveles }\end{array}$ & $\begin{array}{l}4 \text { sótano } \\
13 \\
\text { niveles }\end{array}$ & $\begin{array}{l}3 \text { sótano } \\
14 \text { niveles }\end{array}$ & $\begin{array}{l}3 \text { sótano } \\
10 \text { niveles }\end{array}$ & $\begin{array}{l}3 \text { sótano } \\
10 \text { niveles }\end{array}$ \\
\hline $\begin{array}{l}\text { Área de } \\
\text { construcción }\end{array}$ & $7,640.65 \mathrm{~m}^{2}$ & $\begin{array}{l}8,296.57 \\
\mathrm{~m}^{2}\end{array}$ & $\begin{array}{l}11,481.00 \\
\mathrm{~m}^{2}\end{array}$ & $6,090.33 \mathrm{~m}^{2}$ & $\begin{array}{l}10,073.03 \\
\mathrm{~m}^{2}\end{array}$ \\
\hline Fundaciones & $\begin{array}{l}\text { Zapatas } \\
\text { aisladas con } \\
\text { vigas riostras } \\
\text { y zapatas } \\
\text { corridas }\end{array}$ & $\begin{array}{l}\text { Platea } \\
\text { y vigas } \\
\text { riostras }\end{array}$ & $\begin{array}{l}\text { Platea y } \\
\text { zapatas } \\
\text { corridas }\end{array}$ & $\begin{array}{l}\text { Zapatas } \\
\text { aisladas con } \\
\text { vigas riostras } \\
\text { y zapatas } \\
\text { corridas }\end{array}$ & $\begin{array}{l}\text { Pilotes + } \\
\text { platea }+ \\
\text { zapatas } \\
\text { corridas }\end{array}$ \\
\hline
\end{tabular}




\subsection{Homogenización de las muestras}

En las tablas 5 y 6, mostradas a continuación, se detallan los resultados del nuevo cálculo de malla y acero bajo el método de optimización considerando el acero figurado:

Tabla 5. Cálculo de acero figurado (quintal)

\begin{tabular}{|c|c|c|c|c|c|}
\hline & \multicolumn{5}{|c|}{ No. de muestra } \\
\hline Grupo & $\mathbf{1}$ & $\mathbf{2}$ & $\mathbf{3}$ & $\mathbf{4}$ & $\mathbf{5}$ \\
\hline T1 & 94.20 & 33.38 & 171.35 & 291.70 & 30.60 \\
\hline T2 & $10,966.72$ & $8,008.34$ & $4,176.05$ & $3,993.39$ & $15,390.73$ \\
\hline T3 & $12,349.60$ & $11,216.72$ & $13,501.23$ & $8,705.38$ & $10,709.78$ \\
\hline
\end{tabular}

Tabla 6. Cálculo de malla electrosoldada (quintal)

\begin{tabular}{|c|c|c|c|c|c|}
\hline & \multicolumn{5}{|c|}{ No. de muestra } \\
\hline Grupo & $\mathbf{1}$ & $\mathbf{2}$ & $\mathbf{3}$ & $\mathbf{4}$ & $\mathbf{5}$ \\
\hline T1 & 113.03 & 176.85 & 212.30 & 259.73 & 245.17 \\
\hline T2 & 944.42 & 456.22 & 189.74 & 266.55 & 197.66 \\
\hline T3 & 321.24 & 519.96 & 505.15 & 357.79 & 679.53 \\
\hline
\end{tabular}

\subsection{Porcentaje de costo de acero sobre costos directos}

Para realizar el cálculo del porcentaje de los costos de acero sobre los costos directos, se determinaron, en primer lugar, los costos de los aceros figurados calculados. Dichos costos fueron estimados a partir de la siguiente lista de precios (Tabla 7). 
Tabla 7. Lista de precios acero (Tomado a julio 2019)

\begin{tabular}{|l|c|c|}
\hline \multicolumn{1}{|c|}{ DESCRIPCIÓN } & UD & PRECIO \\
\hline $\begin{array}{l}\text { Acero varilla Grado 40-60 3/8" a 1" x 20 a } \\
30 \text { pies }\end{array}$ & QQ & $\$ 1,981.98$ \\
\hline $\begin{array}{l}\text { Acero varilla Grado 40-60 3/8" a 1" x 20 a } \\
30 \text { pies }\end{array}$ & QQ & $\$ 2,094.59$ \\
\hline $\begin{array}{l}\text { Acero malla electrosoldada D2.3 x D2.3,10 } \\
\text { x 10, Rollo 2.4x40 m. }\end{array}$ & ROLLO & $\$ 12,235.14$ \\
\hline $\begin{array}{l}\text { Acero malla electrosoldada D2.3 x D2.3,15 } \\
\text { x 15, Rollo 2.4x40 m. }\end{array}$ & ROLLO & $\$ 8,162.07$ \\
\hline $\begin{array}{l}\text { Acero malla electrosoldada D2.3 x D2.3, 20 } \\
\text { x 20, Rollo 2.4x40 m }\end{array}$ & ROLLO & $\$ 6,124.99$ \\
\hline $\begin{array}{l}\text { Acero malla electrosoldada D2.5 x D2.5, 10 } \\
\text { x 10, Rollo 2.4x40 m. }\end{array}$ & ROLLO & $\$ 13,327.66$ \\
\hline $\begin{array}{l}\text { Acero malla electrosoldada D2.5 x D2.5, 15 } \\
\text { x 15, Rollo 2.4x40 m. }\end{array}$ & ROLLO & $\$ 8,890.43$ \\
\hline $\begin{array}{l}\text { Acero malla electrosoldada D2.5 x D2.5, 20 } \\
\text { x 20, Rollo 2.4x40 m. }\end{array}$ & ROLLO & $\$ 6,671.80$ \\
\hline $\begin{array}{l}\text { Acero malla electrosoldada D2.7 x D2.7, 10 } \\
\text { x 10, Rollo 2.4x40 m. }\end{array}$ & ROLLO & $\$ 14,344.27$ \\
\hline $\begin{array}{l}\text { Acero malla electrosoldada D2.7 x D2.7, 15 } \\
\text { x 15, Rollo 2.4x40 m. }\end{array}$ & ROLLO & $\$ 9,568.71$ \\
\hline $\begin{array}{l}\text { Acero malla electrosoldada D2.9 x D2.9, 10 } \\
\text { x 10, Rollo 2.4x40 m. }\end{array}$ & ROLLO & $\$ 15,398.30$ \\
\hline $\begin{array}{l}\text { Acero malla electrosoldada D2.9 x D2.9, 15 } \\
\text { x 15, Rollo 2.4x40 m. }\end{array}$ & ROLLO & $\$ 10,271.77$ \\
\hline $\begin{array}{l}\text { Acero malla electrosoldada D2.9 x D2.9, 20 } \\
\text { x 20, Rollo 2.4x40 m. }\end{array}$ & ROLLO \\
\hline
\end{tabular}

En la tabla 8 se muestran los resultados de los costos totales de acero resultantes de multiplicar las cantidades de acero figurado y malla electrosoldada calculadas, por el precio correspondiente, seleccionado a partir de lo indicado en los planos estructurales. 
Tabla 8. Costos totales de acero $(\$ R D)$

\begin{tabular}{|c|c|c|c|c|c|}
\hline & \multicolumn{5}{|c|}{ No. de muestra } \\
\hline Grupo & $\mathbf{1}$ & $\mathbf{2}$ & $\mathbf{3}$ & $\mathbf{4}$ & $\mathbf{5}$ \\
\hline T1 & $\$ 477,829.14$ & $\$ 507,720.77$ & $\$ 885,789.15$ & $\$ 1,258,878.82$ & $\$ 671,052.98$ \\
\hline T2 & $\$ 25,314,668.32$ & $\$ 17,906,460.07$ & $\$ 9,218,011.86$ & $\$ 9,028,209.48$ & $\$ 32,704,082.96$ \\
\hline T3 & $\$ 26,664,662.24$ & $\$ 24,784,907.40$ & $\$ 29,533,268.19$ & $\$ 19,122,202.99$ & $\$ 24,119,079.00$ \\
\hline
\end{tabular}

El otro elemento necesario para el cálculo del porcentaje en cuestión son los costos directos llevados a valor presente, para el cual es necesario el Índice de Costos Directos de la Construcción de Viviendas (ICDV).

Dicho valor presente se calcula de la siguiente manera:

Valor presente costo directo $=\frac{\text { ICDV actual }}{\text { ICDV del mes en que se realizó el proyecto }} \times$ Valor pasado

Para el cálculo de valor presente de los costos directos de las muestras seleccionados se utilizaron los Índice de Costos Directos de la Construcción de Viviendas (ICDV) detallados en las tablas 9 y 10:

Tabla 9. ICDV para junio 2019 (actual)

\begin{tabular}{|c|c|c|c|}
\hline Grupo & T1 & T2 & T3 \\
\hline ICDC & 158.17 & 157.83 & 157.83 \\
\hline
\end{tabular}

Tabla 10. ICDV a la fecha de cada proyecto

\begin{tabular}{|c|c|c|c|c|c|}
\hline & \multicolumn{5}{|c|}{ No. de muestra } \\
\hline Grupo & $\mathbf{1}$ & $\mathbf{2}$ & $\mathbf{3}$ & $\mathbf{4}$ & $\mathbf{5}$ \\
\hline T1 & abr-17 & jun-15 & may-19 & oct-16 & jun-15 \\
\hline Índice 4 niveles & 135.97 & 161.66 & 166.79 & 126.44 & 161.66 \\
\hline T2 & mar-14 & jul-19 & ene-18 & ene-11 & feb-11 \\
\hline Índice 8 + niveles & 186.43 & 174.05 & 157.38 & 187.17 & 187.17 \\
\hline T3 & nov-18 & mar-12 & abr-17 & nov-17 & jul-17 \\
\hline Índice 8 + niveles & 169.45 & 182.72 & 140.91 & 159.16 & 135.01 \\
\hline
\end{tabular}


En la tabla presentada a continuación (tabla 11) se resumen los costos directos de cada proyecto, llevados a valor presente:

Tabla 11. Costos directos llevados a valor presente (julio 2019)-(\$RD)

\begin{tabular}{|c|c|c|c|c|c|}
\hline & \multicolumn{5}{|c|}{ No. de muestra } \\
\hline Grupo & $\mathbf{1}$ & $\mathbf{2}$ & $\mathbf{3}$ & $\mathbf{4}$ & $\mathbf{5}$ \\
\hline T1 & $\$ 7,020,448.85$ & $\$ 4,743,357.99$ & $\$ 6,392,742.33$ & $\$ 15,896,895.87$ & $\$ 6,142,959.17$ \\
\hline T2 & $\$ 312,448,780.99$ & $\$ 226,013,920.34$ & $\$ 57,090,939.71$ & $\$ 62,131,997.13$ & $\$ 137,100,900.95$ \\
\hline T3 & $\$ 142,057,202.49$ & $\$ 173,554,433.81$ & $\$ 487,140,180.46$ & $\$ 115,975,255.79$ & $\$ 260,075,833.49$ \\
\hline
\end{tabular}

Con estos dos insumos se procedió a calcular el porcentaje resultante de los costos de acero (malla y varillas) sobre los costos directos para cada muestra seleccionada. Los resultados de dicho cálculo se detallan a continuación (tabla 12):

Tabla 12. Porcentaje Costo Acero/Costo Directo

\begin{tabular}{|c|c|c|c|c|c|}
\hline & \multicolumn{5}{|c|}{ No. de muestra } \\
\hline Grupo & $\mathbf{1}$ & $\mathbf{2}$ & $\mathbf{3}$ & $\mathbf{4}$ & $\mathbf{5}$ \\
\hline T1 & $6.81 \%$ & $10.70 \%$ & $13.86 \%$ & $7.92 \%$ & $10.92 \%$ \\
\hline T2 & $8.10 \%$ & $7.92 \%$ & $16.15 \%$ & $14.53 \%$ & $23.85 \%$ \\
\hline T3 & $18.77 \%$ & $14.28 \%$ & $6.06 \%$ & $16.49 \%$ & $9.27 \%$ \\
\hline
\end{tabular}

Finalmente, a partir del método PERT se obtuvo una media ponderada para determinar la importancia relativa del costo del acero sobre los costos directos para cada tipo de proyecto. Dichos resultados se detallan a continuación en la tabla 13:

Tabla 13. Resultados media ponderada basados en el Modelo PERT

\begin{tabular}{|c|c|c|c|}
\hline & T1 & T2 & T3 \\
\hline Menor & $6.81 \%$ & $7.92 \%$ & $6.06 \%$ \\
\hline Promedio & $10.04 \%$ & $14.11 \%$ & $13.00 \%$ \\
\hline Mayor & $13.86 \%$ & $23.85 \%$ & $18.77 \%$ \\
\hline $\begin{array}{c}\text { PORCENTAJE COSTO ACERO/ } \\
\text { COSTO DIRECTO }\end{array}$ & $10.14 \%$ & $14.70 \%$ & $12.80 \%$ \\
\hline
\end{tabular}


Estos datos se pueden caracterizar para tener validez dentro de un intervalo de confianza por medio de una prueba estadística. Para esto, a continuación, se presentan los valores representativos para cada grupo acorde la prueba T de Student:

\begin{tabular}{|c|c|c|c|}
\hline \multicolumn{2}{|c|}{ Porcentaje Costo Acero/Costo Directo - T1 } & \multicolumn{2}{c|}{ Porcentaje Costo Acero/Costo Directo - T2 } \\
\hline & & & \\
\hline Promedio & $10.04 \%$ & Promedio & $14.11 \%$ \\
\hline Error estándar & $1.24 \%$ & Error estándar & $2.95 \%$ \\
\hline Mediana & $10.70 \%$ & Mediana & $14.53 \%$ \\
\hline Desviación estándar & $2.77 \%$ & Desviación estándar & $6.59 \%$ \\
\hline $\begin{array}{c}\text { Rango de confianza } \\
(90.0 \%)\end{array}$ & $2.64 \%$ & $\begin{array}{c}\text { Rango de confianza } \\
(90.0 \%)\end{array}$ & $6.28 \%$ \\
\hline
\end{tabular}

\begin{tabular}{|l|r|}
\hline \multicolumn{2}{|c|}{ Porcentaje Costo Acero/Costo Directo - T3 } \\
\hline \multicolumn{2}{|c|}{} \\
\hline Promedio & $12.98 \%$ \\
\hline Error estándar & $2.34 \%$ \\
\hline Mediana & $14.28 \%$ \\
\hline Desviación estándar & $5.22 \%$ \\
\hline Rango de confianza (90.0\%) & $4.98 \%$ \\
\hline
\end{tabular}

Con lo anterior se puede concluir:

- Para los proyectos de vivienda de clase económica los porcentajes de costos de acero sobre los costos directos tiene una media de $10 \%$, el cual con un $90 \%$ de confianza puede oscilar entre $7.64 \%$ y $12.68 \%$.

- Asimismo, para los proyectos de vivienda de clase media los porcentajes de costos de acero sobre los costos directos tiene una media de $14.11 \%$, el cual con un $90 \%$ de confianza puede oscilar entre $7.83 \%$ y $20.39 \%$.

- En los proyectos de vivienda de clase alta los porcentajes de costos de acero sobre los costos directos tiene una media de $12.98 \%$, el cual con un $90 \%$ de confianza puede oscilar $8.00 \%$ y $17.95 \%$. 
En base a estas conclusiones se puede afirmar que los resultados planteados en la tabla 13 tienen validez estadística con un nivel de confianza de $90 \%$.

\subsection{Otros indicadores}

Además de la importancia relativa del costo del acero sobre los costos directos podemos evaluar otras incidencias del acero con respecto a otros valores. En primera instancia, podemos realizar una comparación entre las cantidades de acero figurado de los proyectos contra las cantidades presupuestadas disponibles que consideraron acero liso (por barra).

Tabla 14. Comparativo acero por barra (cantidades presupuestadas) vs acero figurado

\begin{tabular}{|c|c|c|c|}
\hline Muestra & Acero x barra (A) & $\begin{array}{c}\text { Acero figurado } \\
\text { (B) }\end{array}$ & $\begin{array}{c}\text { Diferencia porcentual } \\
\text { (A/B-1) }\end{array}$ \\
\hline T1 M1 & 116.50 & 94.20 & $23.67 \%$ \\
\hline T1 M2 & 39.36 & 33.38 & $17.93 \%$ \\
\hline T1 M3 & 227.21 & 171.35 & $32.60 \%$ \\
\hline T3 M2 & $13,572.23$ & $11,216.72$ & $21.00 \%$ \\
\hline T3 M4 & $11,067.15$ & $8,705.38$ & $27.13 \%$ \\
\hline T3 M5 & $13,648.90$ & $10,709.78$ & $27.44 \%$ \\
\hline
\end{tabular}

Se puede observar claramente en la tabla anterior que las cantidades presupuestadas superan a las de acero figurado en todos los casos. Existe una diferencia porcentual entre ambas cuantificaciones que promedia un $24.96 \%$.

Si extrapolamos esta evaluación a términos de costos, utilizando el listado de precios de la tabla 7 , obtendríamos los siguientes resultados: 
Tabla 15. Comparativo de costos de acero por barra vs acero figurado

\begin{tabular}{|c|c|c|c|}
\hline Muestra & $\begin{array}{c}\text { Costo total Acero } \mathbf{x} \\
\text { barra (RD\$) }\end{array}$ & $\begin{array}{c}\text { Costo total Acero } \\
\text { figurado (RD\$) }\end{array}$ & $\begin{array}{c}\text { Diferencia Acero } \\
\text { figurado/Acero } \mathbf{x} \text { barra }\end{array}$ \\
\hline T1 M1 & $\$ 230,900.90$ & $\$ 197,313.10$ & $-17.02 \%$ \\
\hline T1 M2 & $\$ 78,012.77$ & $\$ 69,908.98$ & $-11.59 \%$ \\
\hline T1 M3 & $\$ 450,334.64$ & $\$ 358,908.78$ & $-25.47 \%$ \\
\hline T3 M2 & $\$ 26,899,920.88$ & $\$ 23,494,483.87$ & $-14.49 \%$ \\
\hline T3 M4 & $\$ 21,934,889.51$ & $\$ 18,234,240.62$ & $-20.30 \%$ \\
\hline T3 M5 & $\$ 27,051,876.23$ & $\$ 22,432,647.60$ & $-20.59 \%$ \\
\hline
\end{tabular}

Acorde a los resultados anteriores, se puede concluir que la consideración de trabajar con acero figurado para proyectos de vivienda económica representa un ahorro promedio de un $18.03 \%$ y para proyectos de vivienda de clase alta un $18.46 \%$. En adición, otro indicador que cabe destacar es la relación entre área de construcción y cantidad de acero figurado calculado (Relación $q q / \mathrm{m}^{2}$ ). Es a partir del análisis de este indicador que podemos inferir el intervalo en el cual puede ubicarse la relación de acero por $\mathrm{m}^{2}$ a utilizar para cada tipo de vivienda.

A continuación, se detallan, por tipo de vivienda, los resultados de la Relación $q q / \mathrm{m}^{2}$, junto a los indicadores estadísticos de lugar necesarios para caracterizar la data y calcular el intervalo en que puede oscilar este valor para un rango de confianza de un $90 \%$.

\subsubsection{Tipo 1 (Proyectos de viviendas de clase económica):}

Tabla 16. Relación $\mathrm{qq} / \mathrm{m}^{2}$ proyectos de viviendas Tipo 1

\begin{tabular}{|c|c|c|c|c|c|}
\hline Muestra & $\begin{array}{c}\text { Área de } \\
\text { construcción } \\
\left.\mathbf{( M}^{2}\right)\end{array}$ & $\begin{array}{c}\text { Acero } \\
\mathbf{( q q})\end{array}$ & $\begin{array}{c}\text { Relación qq/ } \\
\mathbf{M}^{2} \text { (Acero) }\end{array}$ & $\begin{array}{c}\text { Malla } \\
\mathbf{( q q )}\end{array}$ & $\begin{array}{c}\text { Relación qq/M } \\
\text { (Malla) }\end{array}$ \\
\hline T1 M1 & 479.68 & 94.20 & 0.20 & 113.03 & 0.24 \\
\hline T1 M2 & 398.27 & 33.38 & 0.08 & 176.85 & 0.44 \\
\hline T1 M3 & 611.72 & 171.35 & 0.28 & 212.30 & 0.35 \\
\hline T1 M4 & 987.13 & 291.70 & 0.30 & 259.73 & 0.26 \\
\hline T1 M5 & 597.58 & 30.60 & 0.05 & 245.17 & 0.41 \\
\hline
\end{tabular}


Tabla 17. Análisis estadístico relación qq/m2 acero y malla (Grupo T1)

\begin{tabular}{|c|c|c|c|}
\hline \multicolumn{2}{|c|}{$\begin{array}{c}\text { Análisis Relación qq/M } \\
\text { Grupo T1 }\end{array}$} & \multicolumn{2}{c|}{$\begin{array}{c}\text { Análisis Relación } \\
\text { Grupo T1 }\end{array}$} \\
\hline Promedio & 0.181 & Promedio & 0.340 \\
\hline Error estándar & 0.050 & Error estándar & 0.040 \\
\hline Mediana & 0.196 & Mediana & 0.347 \\
\hline $\begin{array}{c}\text { Desviación } \\
\text { estándar }\end{array}$ & 0.111 & $\begin{array}{c}\text { Desviación } \\
\text { estándar }\end{array}$ & 0.090 \\
\hline $\begin{array}{c}\text { Rango de } \\
\text { confianza (90.0\%) }\end{array}$ & 0.106 & $\begin{array}{c}\text { Rango de } \\
\text { confianza (90.0\%) }\end{array}$ & 0.086 \\
\hline
\end{tabular}

A partir de la información presentada en las tablas anteriores se puede concluir que para los proyectos de vivienda de clase económica la relación de acero qq $/ \mathrm{m}^{2}$ tiene una media de $0.181 \mathrm{qq} / \mathrm{m}^{2}$, la cual, con un $90 \%$ de confianza, puede oscilar entre 0.075 y $0.287 \mathrm{qq} / \mathrm{m}^{2}$. Mientras que para la relación de malla qq $/ \mathrm{m}^{2}$ se obtuvo una media de $0.340 \mathrm{qq} / \mathrm{m}^{2}$, la cual, con un $90 \%$ de confianza, puede oscilar entre 0.254 y $0.426 \mathrm{qq} / \mathrm{m}^{2}$.

\subsubsection{Tipo 2 (Proyectos de viviendas de clase media)}

Tabla 18. Relación $\mathrm{qq} / \mathrm{m}^{2}$ proyectos de viviendas Tipo 2

\begin{tabular}{|c|c|c|c|c|c|}
\hline Muestra & $\begin{array}{c}\text { Área de } \\
\text { construcción }\left(\mathbf{M}^{2}\right)\end{array}$ & $\begin{array}{c}\text { Acero } \\
\text { (qq) }\end{array}$ & $\begin{array}{c}\text { Relación qq/M } \\
\text { (Acero) }\end{array}$ & $\begin{array}{c}\text { Malla } \\
\text { (qq) }\end{array}$ & $\begin{array}{c}\text { Relación qq/M } \\
\text { (Malla) }\end{array}$ \\
\hline T2 M1 & $16,393.10$ & $10,966.72$ & 0.67 & 944.42 & 0.06 \\
\hline T2 M2 & $6,567.78$ & $8,008.34$ & 1.22 & 456.22 & 0.07 \\
\hline T2 M3 & $3,483.03$ & $4,176.05$ & 1.20 & 189.74 & 0.05 \\
\hline T2 M4 & $5,051.30$ & $3,993.39$ & 0.79 & 266.55 & 0.05 \\
\hline T2 M5 & $6,058.36$ & $15,390.73$ & 2.54 & 197.66 & 0.03 \\
\hline
\end{tabular}


Tabla 19. Análisis estadístico relación $\mathrm{qq} / \mathrm{m}^{2}$ acero y malla (Grupo T2)

\begin{tabular}{|c|c|c|c|}
\hline \multicolumn{2}{|l|}{$\begin{array}{c}\text { Análisis Relación } q q / M^{2} \text { Acero - } \\
\text { Grupo T2 }\end{array}$} & \multicolumn{2}{|c|}{$\begin{array}{c}\text { Análisis Relación } q q / M^{2} \text { Malla - } \\
\text { Grupo T2 }\end{array}$} \\
\hline Promedio & 1.284 & Promedio & 0.053 \\
\hline Error estándar & 0.333 & Error estándar & 0.006 \\
\hline Mediana & 1.199 & Mediana & 0.054 \\
\hline Desviación estándar & 0.744 & Desviación estándar & 0.013 \\
\hline $\begin{array}{l}\text { Rango de confianza } \\
(90.0 \%)\end{array}$ & 0.709 & $\begin{array}{l}\text { Rango de confianza } \\
(90.0 \%)\end{array}$ & 0.013 \\
\hline
\end{tabular}

A partir de la información presentada en las tablas anteriores se puede concluir que para los proyectos de vivienda de clase media la relación de acero $\mathrm{qq} / \mathrm{m}^{2}$ tiene una media de $1.284 \mathrm{qq} / \mathrm{m}^{2}$, la cual, con un $90 \%$ de confianza puede oscilar entre 0.575 y $1.993 \mathrm{qq} / \mathrm{m}^{2}$. Mientras que para la relación de malla qq $/ \mathrm{m}^{2}$ se obtuvo una media de $0.053 \mathrm{qq} / \mathrm{m}^{2}$, la cual, con un $90 \%$ de confianza, puede oscilar entre 0.040 y $0.066 \mathrm{qq} / \mathrm{m}^{2}$.

\subsubsection{Tipo 3 (Proyectos de viviendas de clase alta)}

Tabla 20. Relación $\mathrm{qq} / \mathrm{m}^{2}$ proyectos de viviendas Tipo 3

\begin{tabular}{|c|c|c|c|c|c|}
\hline Muestra & $\begin{array}{c}\text { Área de construcción } \\
\left(\mathbf{M}^{2}\right)\end{array}$ & Acero (qq) & $\begin{array}{c}\text { Relación } \\
\mathbf{q q} / \mathbf{M}^{\mathbf{2}}\end{array}$ & $\begin{array}{c}\text { Malla } \\
\mathbf{( q q})\end{array}$ & $\begin{array}{c}\text { Relación } \\
\mathbf{q q} / \mathbf{M}^{\mathbf{2}}\end{array}$ \\
\hline T3 M1 & $7,640.65$ & $12,349.60$ & 1.62 & 321.24 & 0.04 \\
\hline T3 M2 & $8,296.57$ & $11,216.72$ & 1.35 & 519.96 & 0.06 \\
\hline T3 M3 & $11,481.00$ & $13,501.23$ & 1.18 & 505.15 & 0.04 \\
\hline T3 M4 & $6,090.33$ & $8,705.38$ & 1.43 & 357.79 & 0.06 \\
\hline T3 M5 & $10,073.03$ & $10,709.78$ & 1.06 & 679.53 & 0.07 \\
\hline
\end{tabular}


Tabla 21. Análisis estadístico relación $\mathrm{qq} / \mathrm{m}^{2}$ acero y malla (Grupo T3)

\begin{tabular}{lrlr}
\hline Análisis Relación & $\mathbf{q} / \mathbf{M}^{2}$ Acero - Grupo T3 & Análisis Relación qq/M Malla - Grupo T3 \\
\hline Promedio & 1.327 & Promedio & 0.055 \\
Error estándar & 0.097 & Error estándar & 0.005 \\
Mediana & 1.352 & Mediana & 0.059 \\
Desviación estándar & 0.216 & Desviación estándar & 0.011 \\
Rango de confianza (90.0\%) & 0.206 & Rango de confianza $(90.0 \%)$ & 0.011 \\
\hline
\end{tabular}

A partir de la información presentada en las tablas anteriores se puede concluir que para los proyectos de vivienda de clase alta la relación de acero $\mathrm{qq} / \mathrm{m}^{2}$ tiene una media de $1.327 \mathrm{qq} / \mathrm{m}^{2}$, la cual, con un $90 \%$ de confianza, puede oscilar entre 1.121 y $1.533 \mathrm{qq} / \mathrm{m}^{2}$. Mientras que para la relación de malla qq $/ \mathrm{m}^{2}$ se obtuvo una media de $0.055 \mathrm{qq} / \mathrm{m}^{2}$, la cual, con un $90 \%$ de confianza, puede oscilar entre 0.044 y $0.066 \mathrm{qq} / \mathrm{m}^{2}$.

\section{Conclusiones}

En busca de determinar la proporción de costo total del acero dentro de los costos directos de proyectos de viviendas clase económica, media y alta en la provincia de Santo Domingo, República Dominicana, para el año 2019, utilizamos la estrategia metodológica de "estudio de caso" para dirigirnos de lo particular a lo general evaluando un conjunto de planos y presupuestos de proyectos, filtrados por ciertas características, que encajarán dentro de los tres grupos principales.

Para cada uno de estos grupos se recalculó la cantidad total de malla electrosoldada y de acero, considerando desperdicio cero (acero figurado), para luego determinar el valor presente del costo total de los mismos y los costos directos totales de las muestras utilizando el Índice de Costos Directos de la Construcción de Viviendas (ICDV) que elabora la ONE con apoyo de ACOPROVI. Estos valores se utilizaron para cumplir el objetivo determinar la proporción de costo total del acero dentro de los costos directos de proyectos de viviendas clase económica, media y alta 
en la provincia de Santo Domingo, para el año 2019. Ver tabla 11 para referencia.

Fuera de esta proporción de costo total del acero dentro de los costos directos de proyectos, se evaluaron estadísticamente otros indicadores para medir la relación del total de acero sobre las siguientes variables:

- Acero figurado: con relación a las cantidades totales utilizadas en la muestra, existe una diferencia porcentual entre ambas cuantificaciones que promedia un $24.96 \%$ siendo el acero figurado menor que el acero listo. En costo, la consideración de trabajar con acero figurado para proyectos de vivienda económica representa un ahorro promedio de un $18.03 \%$ y para proyectos de vivienda de clase alta un $18.46 \%$.

- Área de construcción: para este caso, en los proyectos de vivienda de clase alta la relación de acero $\mathrm{qq} / \mathrm{m}^{2}$ tiene una media de 1.327 $\mathrm{qq} / \mathrm{m}^{2}$. Mientras que para la relación de malla $\mathrm{qq} / \mathrm{m}^{2}$ se obtuvo una media de $0.055 \mathrm{qq} / \mathrm{m}^{2}$.

Para complementar estos resultados, y que sean de mejor provecho para la industria de la construcción, consideramos importante evaluar en futuras investigaciones la reducción en tiempo de las labores de acero en obra y la calidad de su colocación al recibir el acero figurado in situ, así como su impacto económico en los costos directos e indirectos de proyectos. Asimismo, la habilitación de acero en fábrica (acero figurado) que permitiría a los diseñadores estructurales utilizar diámetros no comerciales en barras lisas para la optimización de sus diseños.

\section{Referencias}

Banco Central de la República Dominicana. (14 de abril de 2019). Producto Interno Bruto (PIB), Trimestral 2007-2018. Recuperado de Transparencias: https://www.bancentral.gov.do/transparencias

Bernal, C. A. (2010). Metodología de la investigación. Administración, económia, humanidades y ciencias sociales. (Tercera Ed.). Colombia: Pearson Educación. 
División Investigación ONE. (2010). El déficit habitacional en República Dominicana: Panoramaactualizado de los requerimientos habitacionales. República Dominicana: Oficina Nacional de Estadística.

Editorial Construir. (14 deabril de2019). Construcción deviviendas en República Dominicana crece durante el primer trimestre de 2018. Recuperado de http://revistaconstruir.com/construccion-de-viviendas-en-republicadominicana-crece-durante-el-primer-trimestre-de-2018/

Hernandéz Sampieri, R. (2014). Metodología de la Investigación. México: McGraw-Hill.

Kinnear, T., \& Taylor, J. (1993). Investigación de Mercados. México: McGraw-Hill.

Ministerio de Economía, Planificación y Desarrollo (MEPyD). (2018). Metodología para la medición del deficit habitacional en República Dominicana. Recuperado de: http://economia.gob.do/publicaciones/ metodologia-para-la-medicion-del-deficit-habitacional-enrepublica-dominicana-2018

Oficina Nacional de Estadística (ONE). (2019). Estudio de Oferta de Edificaciones. Departamento de Estadísticas Económicas. Santo Domingo, República Dominicana: Oficina Nacional de Estadística.

Oficina Nacional de Estadística. (2019). Indice de Costos Directos de la Construcción de Viviendas (ICDV). Santo Domingo República Dominicana. Recuperado de https://www.one.gob.do/precios-ycostos/icdv

Paz, K. (2007). Media Aritmética Simple. (U. R. Landivar, Ed.) Boletín Electrónico. Recuperado de http://www.fsalazar.bizland.com/LANDIVAR/ING-PRIMERO/boletin07/URL_07_BAS01.pdf

Rodríguez, T., \& Abbott, M. (2008). Análisis comparativo entre el método de asignación directa y el de optimización para cuantificación del refuerzo levantado en los apoyo en placas sólidas y su efecto en la generación de desperdicios. Ciencia y Sociedad, 33(4), 572-594. 